\title{
A calibration of the small solar radiotelescope using
}

\section{a new moon}

\begin{abstract}
Background: Defining the solar brightness temperature at centimetre wavelengths has always been challenging. One of the main reasons has been the lack of a proper calibration source. We carried out observations at $2.7 \mathrm{~cm}(11.2 \mathrm{GHz})$ by using the New Moon for the solar brightness temperature calibration. The solar and Moon observations were made using the 1.8-meter radiotelescope operated by the Aalto University Metsähovi Radio Observatory in Finland. In this article, we present our method for defining the brightness temperature of the quiet-Sun level (QSL). Based on these observations, we found $11500 \mathrm{~K} \pm 1500 \mathrm{~K}$ to be the mean value for the QSL temperature. This value is quite similar which was reported earlier.
\end{abstract}

Volume 3 Issue 3 - 2019

\author{
Juha Kallunki \\ Metsähovi Radio Observatory, Aalto University, Finland
}

Correspondence: Juha Kallunki, Metsähovi Radio Observatory, Aalto University, Metsähovintie I 1402540 Kylmälä, Finland, Tel +358 29 4424852, Email juha.kallunki@aalto.fi

Received: February 13,2019 | Published: May 10, 2019

Keywords: radiotelescope, radio observations, solar brightness temperature, quietSun level (QSL), lunar brightness temperature

\section{Introduction}

Defining the solar brightness temperature at centimetre has always been difficult. One of the main reasons has been the lack of a proper calibration source. The New Moon has discovered to be a stabile and a sufficient powerful calibration source. The most recent studies are made by. ${ }^{1-3}$ The solar brightness temperature calibration makes possible that all the other detected solar radio burst can be scaled more reliable to the quiet-Sun level (QSL) temperature. The emission at 11.2 $\mathrm{GHz}$ originates either in the upper chromosphere or in the transition region. More accurately defined brightness temperature value of the QSL could also explain the origin of the emission more carefully. The main purpose of this work is to find calibrated solar brightness temperature at $11.2 \mathrm{GHz}$. In addition, we want to study the beam shape of the radiotelescope. For proper radiotelescope calibration, it is useful to know the beam width, shape and possible beam side lobes as well. This work is continuation to study done by ${ }^{1}$ and the methods used in this study are consistent with them.

\section{Instrumentation}

Metsähovi MRO-1.8, located at Metsähovi Radio Observatory (MRO), Aalto University (Helsinki Region, Finland, GPS coordinates: $\mathrm{N} 60^{\circ} 13.04^{\prime}, \mathrm{E} 24^{\circ} 23.35^{\prime}$ ), is a radio telescope with a $1.8 \mathrm{~m}$ dish diameter dedicated for continuous solar observations. The operation frequency range of the radiotelescope is between 10.7 and 11.7 $\mathrm{GHz}$. The front-end of the receiving unit is a low-cost commercial component, a low-noise block down converter (LNB), which converts radio frequency to the intermediate frequency $(950-1950 \mathrm{MHz})$. And finally, the total power of the intermediate frequency band will be sampled and recorded. The telescope's system temperature is higher than $300 \mathrm{~K}$. The full documentation of MRO-1.8 radiotelescope can be found from. ${ }^{4-6}$ In Figure 1, MRO-1.8 radiotelescope is shown. MRO1.8 radiotelescope has been operational since 2001 and it has detected more than a hundred solar radio bursts. ${ }^{5}$ Besides studying versatility solar radio burst properties, ${ }^{5,7}$ for instance oscillation features have been studied using MRO-1.8 .

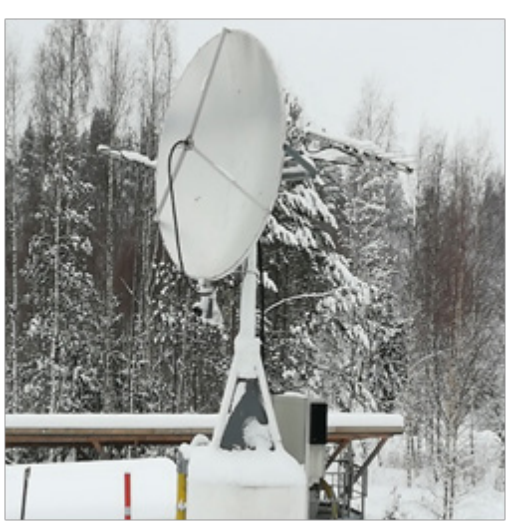

Figure I A photograph of MRO-I.8 radiotelescope. Two log-periodic antennae attached to both sides of the dish observe the Sun at the decimeter wavelengths.

\section{Observations}

The calibration observations were made between winter 2018 and spring 2019. Totally six consecutive Sun-Moon-observations were made; the lunar map was observed at first and immediately after this, solar map was made. The observations were raster scan maps (30 sweeps) over the Sun (Figure 2) and the Moon (Figure 3) disks. The radiotelescope is not designed for scanning observations, thus it took ca. 30 minutes to make one raster map. The radiotelescope is designed to track Sun continuously. The full raster scan map was made with the size of 3 degrees $x 3$ degrees. A single step in the raster scan map was 300 millidegree, thus one raster map consists on 441 measurement points. The step size is roughly one third of the full beam size. In Table 1, a summary of all observations is presented. The consecutive Sun-Moon-map combinations are made similar elevation ranges $( \pm 5$ degrees). Observation days were selected so that the activity of the Sun at $11.2 \mathrm{GHz}$ was low. A cosine correction is applied to the results to eliminate the effect of the changing elevation during each raster scan map. The previous study ${ }^{1}$ was made at $37 \mathrm{GHz}$. That frequency 
is more sensitive for the prevailing atmospheric (weather) conditions than 11.2 GHz. MRO-1.8 radiotelescope has no protective radome.

Table I Summary of Sun-Moon observations: date, offset (in days) from the New Moon, the calculated Moon brightness temperature, the Sun brightness temperature and the activity status of the Sun

\begin{tabular}{lllll}
\hline Date & $\begin{array}{l}\text { Offset } \\
\text { (day) }\end{array}$ & $T_{\text {moon }}$ & $T_{\text {sun }}$ & Status of the Sun \\
\hline I2/13/2018 & 6 & 232.8 & 11347 & Very minor activity \\
$2 / 4 / 2019$ & 0 & 232.3 & 11882 & Small activity \\
$2 / 5 / 2019$ & 1 & 231 & 10831 & Small activity \\
$2019-02-06(1)$ & 2 & 230.2 & 12863 & Small activity \\
$2019-02-06(2)$ & 2 & 230.2 & 10032 & Small activity \\
$2 / 8 / 2019$ & 4 & 230.4 & 12272 & Small activity \\
\hline
\end{tabular}

Defining the solar brightness temperature at II.2 $\mathbf{G H z}$

In Figure 2, the most upper read line indicates the solar maximum intensity. Actually, this intensity is a sum of solar and background (sky) intensities $\left(I_{\text {sun }}+I_{\text {background }}\right)$. The lowest read line indicates only the background (sky) intensity (I background), thus the difference between these values is a real solar intensity $\left(I_{\text {sun }}\right)$. Similar deduction is applying for the Moon, Figure $2\left(I_{\text {moon }}\right)$. The maximum and minimum intensities were also verified from the raster maps (upper plots in Figure 2 \& Figure 3). The maximum intensity was typically found from the middle sweep (lower plots in Figure $2 \&$ Figure 3) of the raster scan map. If the pointing of the telescope is not perfect, then the middle sweep will not be fully reliable. That is why the raster scan maps were made.

After that, the Sun-Moon-ratio $\left(r_{\text {sun moon }}\right)$ can be calculated as $I_{\text {sun }} / I_{\text {moon }}$. The Moon brightness temperature $T_{\text {moon }}(v)$ is described as ${ }^{2}$

$$
\operatorname{Tmoon}(v)=T 0(v)+T 1(v) \cos (\omega t-\xi(v))
$$

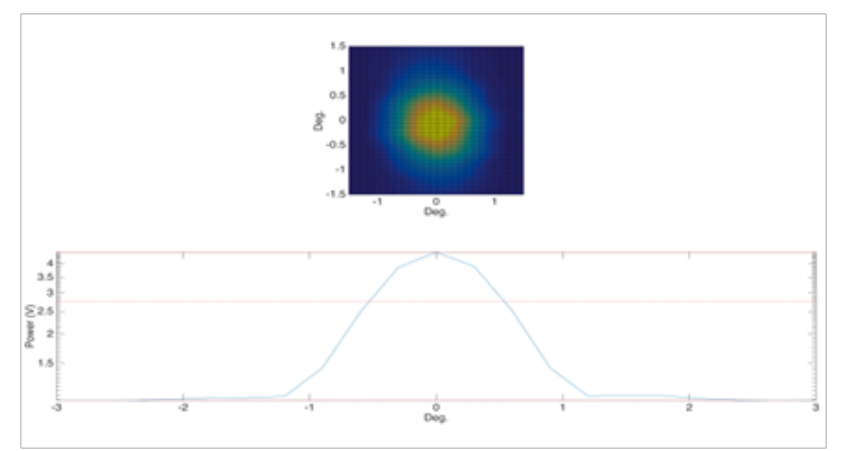

Figure 2 In upper plot, a raster scan map of the Sun and in lower plot, the middle sweep (I5 out of 30) over the solar disk. In upper plot, the yellow colour indicates high intensity and the blue colour indicates low intensity (=sky intensity).

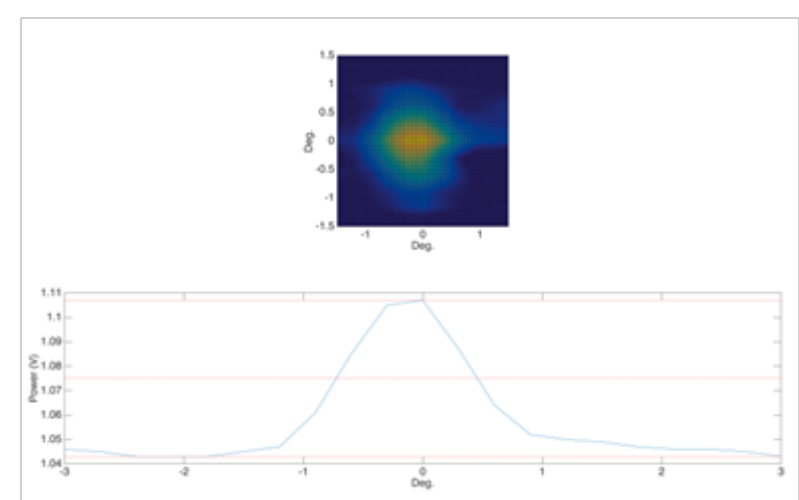

Figure 3 In upper plot, a raster scan map of the Moon and in lower plot, the middle sweep (I5 out of 30) over the lunar disk. In upper plot, the yellow colour indicates high intensity and the blue colour indicates low intensity (=sky intensity).

where the mean brightness $\left(T_{0}(v)\right)$ temperature over a lunation at $11,2 \mathrm{GHz}$ is $218 \mathrm{~K}$. The amplitude of the 29.3-d first harmonic $\left(T_{l}(v)\right)$ is $12 \mathrm{~K}$. And the phase shift $(v)$ of this harmonic as measured from the time of the New Moon is $35^{\circ} . \omega$ is the angular frequency of the lunar cycle $\left(12.26^{\circ}\right.$ per day). The Moon brightness temperatures $\left(T_{\text {moon }}\right)$ during the observations are summarized in Table 1. Finally, the solar brightness temperature $\left(T_{\text {sun }}\right)$ can be defined as $T_{\text {moon }} x r_{\text {sun moon }}$ . This value is the quiet Sun level (QSL) at $11.2 \mathrm{GHz}$. The observed solar brightness temperatures are also presented in Table 1 . The values for all the observing sessions are the mean QSL brightness temperature $11538 \mathrm{~K}$, the minimum QSL temperature $10032 \mathrm{~K}$ and the maximum QSL temperature $12863 \mathrm{~K}$. From our measurements, we conclude that the solar brightness temperature at $8 \mathrm{~mm}$ is $11500 \mathrm{~K} \pm 1500 \mathrm{~K}$.

\section{Beam pattern}

The full width half power (FWHP) on the radiotelescope is defined as $1.02 \times \lambda / \mathrm{D}$, where $\lambda$ is the used wavelength and $D$ is the diameter of the radiotelescope's main reflector'. Thus at $11.2 \mathrm{GHz}$ FWHP is 0.87 degree. This is an ideal, the theoretical beam width. Our measurement, on the basis on solar scans over the solar equator, showed that the beam width is $1.09^{\circ}$. The difference between these values is clear; the radiotelescope's design is not ideal. The middle red line in Figure 2 and Figure 3 indicates FWHP. In addition, small side lobes are detected distance of 1.5 degree from the top of the telescope's main lobe. However, the side lobes are only $2 \%$ higher than the sky intensity, which can be seen in Figure 1 (position of the weak side lobe is marked as a blue arrow). The Moon is too weak source to study side lobe effect. We cannot see any clear indication on side lobes in Figure 3 (lower plot). We can conclude that the feed and other telescope mechanics are designed properly. The raster scan map was made with size of 3 degrees $\times 3$ degrees, thus if there are side lobes with the distance more than 3 degrees from the main lobe, we did not notice those.

\section{Conclusion}

Our observations showed that the QSL temperature value at 11.2 $\mathrm{GHz}$ is $11500 \mathrm{~K} \pm 1500 \mathrm{~K}$. This value matches the values that have mostly been used in the literature so far, $12000^{5} \mathrm{~K}$ and $12200 \mathrm{~K}^{2}$. The 
defined QSL temperature is so low that we can assume that the emission originates from the upper chromosphere more than the lower part of the transition region. MRO-1 radiotelescope has been previously used only for the solar observations. This was the first attempt to observe other source than the Sun. The telescope's system sensitivity was also sufficient for the lunar observations. Maybe other stabile radio sources could also be used for the calibration purposes e.g. Jupiter. This should also be tested in the nearest future. The possible error sources are the fact that observations are made at relatively low elevations $(<20$ degrees) angles. The radiotelescope is not designed for raster scan mapping and single map observations took more than 30 minutes. On that period of time, atmospheric condition may vary. In the future, we have to study if the single sweeps (middle sweep) could be decent for the calibration analysis. We will start the calibration observations on a regular basis (monthly) near the occasions of New Moon. The telescope is dedicated only for solar observations, thus the time for the calibration observation can be arranged easily. The calibration should be made times when Sun's activity status is low; high solar activity may distort the observation results. In addition, the weather conditions should be reasonable during the calibration observation sessions. The MRO-1.8 radiotelescope has also calibration (noise) diode injection possibility. The noise source can be controlled remotely. We will try to find a solution to use more effectively noise source, and could we use it also in calibration purposes (brightness temperature definition). One of the main results is that we got a confirmation that the calibration method for defining QSL brightness temperature is functional even if the radiotelescope is smaller than in the previous study, ${ }^{1}$ sensitivity is lower and the observation frequency range is different.

\section{Acknowledgments}

None.

\section{Conflicts of interests}

Authors declare that there is no conflict of interest.

\section{References}

1. Kallunki J, Tornikoski M. Measurements of the Quiet-Sun Level Brightness Temperature at $8 \mathrm{~mm}$. Solar Physics. 2018;293(11):8.

2. Hafez, Yaser A, Trojan, et al. A radio determination of the time of the New Moon. Monthly Notices of the Royal Astronomical Society. 2014;439(3):2271-2280.

3. Iwai, Kazumasa, Shimojo, et al. The Brightness Temperature of the Quiet Solar Chromosphere at 2.6 mm. Solar Physics. 2017;292(1):18.

4. Uunila, Minttu, Kallunki, et al. Reliability of 1.8-meter solar radio telescope at Metsähovi Radio Observatory for long-term solar monitoring. Astrophysics and Space Science. 2015;359:6.

5. Kallunki Juha, Uunila Minttu. Total Solar Flux Intensity at $11.2 \mathrm{GHz}$ as an Indicator of Solar Activity and Cyclicity. International Journal of Astronomy and Astrophysics. 2014;4(3):437-444

6. Kallunki J, Tornikoski M, Tammi J, et al. Forty years of solar radio observations at Metsähovi Radio Observatory. Astronomische Nachrichten. 2018;339(204):204-211

7. Kallunki J, Uunila M, McKay-Bukowski D. Multi-frequency solar observations at Metsähovi Radio Observatory and KAIRA. Astronomische Nachrichten. 2015;336(6):566.

8. Khodachenko ML, Kislyakova KG, Zaqarashvili TV, et al. Possible manifestation of large-scale transverse oscillations of coronal loops in solar microwave emission. Astronomy and Astrophysics. 2011;525:id.A105:4.

9. Marr JM, Snell RL, Kurtz ES. Fundamentals of Radio Astronomy: Observational Methods. $1^{s t}$ edn. CRC Press. 2005. 